\title{
Profile Likelihood Estimation of the
} Correlation Coefficient in the Presence of Left, Right or Interval Censoring and Missing Data

\author{
by Yanming Li, Brenda W. Gillespie, Kerby Shedden, John A. Gillespie
}

\begin{abstract}
We discuss implementation of a profile likelihood method for estimating a Pearson correlation coefficient from bivariate data with censoring and/or missing values. The method is implemented in an $R$ package clikcorr which calculates maximum likelihood estimates of the correlation coefficient when the data are modeled with either a Gaussian or a Student $t$-distribution, in the presence of left, right, or interval censored and/or missing data. The $\mathrm{R}$ package includes functions for conducting inference and also provides graphical functions for visualizing the censored data scatter plot and profile log likelihood function. The performance of clikcorr in a variety of circumstances is evaluated through extensive simulation studies. We illustrate the package using two dioxin exposure datasets.
\end{abstract}

\section{Introduction}

Partially observed data present a challenge in statistical estimation. Simple approaches like complete case analysis allow traditional estimators to be used, but sacrifice precision and may introduce bias. More sophisticated approaches that incorporate information from partially observed cases have the potential to achieve greater power, but can be much more difficult to implement. As a case in point, the Pearson correlation coefficient is easily estimated by the familiar moment-based formula,

$$
r=\frac{\sum_{i=1}^{n}\left(x_{i}-\bar{x}\right)\left(y_{i}-\bar{y}\right)}{\left[\sum_{i=1}^{n}\left(x_{i}-\bar{x}\right)^{2} \sum_{i=1}^{n}\left(y_{i}-\bar{y}\right)^{2}\right]^{1 / 2}}
$$

and under certain conditions, uncertainty in the estimate can be assessed using Fisher's variance stabilizing transformation (Fisher, 1915). However, it is not obvious how this approach can be extended to accommodate partially observed data.

Partially observed bivariate data $(x, y)$ can take several forms. If either $x$ or $y$ is completely missing, the observation provides no direct information about the correlation coefficient, but is informative about the marginal distributions. If $x$ and/or $y$ is censored, but neither is completely missing, then the observation provides information about the correlation parameter as well as about the marginal distributions.

Here we discuss a likelihood-based method for estimating the correlation coefficient from partiallyobserved bivariate data, and present an R package implementing the method, clikcorr ("Censored data likelihood based correlation estimation") (Li et al., 2016). clikcorr calculates maximum likelihood estimates of the parameters in a model, in particular the correlation coefficient, in the presence of left, right, and interval censored data and missing values. clikcorr also has functions to help visualize bivariate datasets that contain censored and/or missing values.

The package emphasizes the role of the profile likelihood function for the correlation parameter. It provides functions to visualize the profile likelihood function, and uses likelihood ratio test inversion to provide confidence intervals for the correlation coefficient. This circumvents difficulties that arise when using Wald-type confidence intervals for a parameter that lies in a bounded domain, and that may have an asymmetric sampling distribution.

This project was motivated by the need to calculate correlation coefficients with left-censored chemical assay data. Such data often arise in the context of toxicology, chemistry, environmental science and medical laboratory applications, where some measurements fall below a limit of detection (LOD) (Jaspers et al., 2013). The limit of detection must be known or approximated. Estimating the correlation coefficient from bivariate censored data has been studied (Li et al., 2005), but issues in both methodology and software remain.

The paper is organized as follows. Section 24.1 develops model-based frameworks for correlation estimation based on Gaussian and Student-t models. Section 24.2 describes clikcorr, including the data input format, the output format, and program features such as the plotting functions. Section 24.3 presents simulations to address the performance of clikcorr under a range of scenarios, focusing on run time and coverage probability of the confidence intervals. In Section 24.4 we use clikcorr to 
analyze dioxin data from both Jaspers et al. (2013) and the National Health and Nutrition Examination Survey (NHANES). Some further details on using the R package clikcorr are provided in Section 24.5

\section{Models for correlation parameter estimation}

Working in a model-based framework of a bivariate distribution with a correlation parameter opens up several paths for handling partially observed data. A likelihood for the observed data can be calculated by integrating a "complete data" likelihood over all data configurations that are consistent with the observed data. The resulting "observed data likelihood" is seen as preserving full information from the data, and can be used with any standard likelihood-based inference approach, including maximum likelihood (ML) estimation, Wald-type inference, likelihood ratio testing, and profile likelihood analysis.

The bivariate Gaussian distribution is a natural starting point for model-based estimation of a correlation coefficient. The distribution has five parameters: two marginal means $\left(\mu_{X}, \mu_{Y}\right)$, two marginal standard deviations $\left(\sigma_{X}, \sigma_{Y}\right)$, and the correlation coefficient $\rho_{X Y}$. The joint density function of the bivariate Gaussian distribution for standardized data $\left(\mu_{x}=\mu_{y}=0, \sigma_{x}=\sigma_{y}=1\right)$ is

$$
f_{0}(x, y)=\frac{1}{2 \pi \sqrt{1-\rho_{X Y}^{2}}} \exp \left(-\frac{x^{2}+y^{2}-2 \rho_{X Y} x y}{2\left(1-\rho_{X Y}^{2}\right)}\right) .
$$

When the data are complete, the maximum likelihood estimates of the parameters in the bivariate Gaussian distribution are the sample means $(\bar{x}$ and $\bar{y})$, the sample standard deviations (scaled by $\sqrt{(n-1) / n)}$, and the sample correlation coefficient, respectively. These are all closed-form estimators, with the correlation coefficient estimator $r$ given by (1).

The fact that the Gaussian distribution maximum likelihood estimates coincide with the standard method of moments estimates implies some desirable properties. In particular, in the complete data setting, the Gaussian ML estimates will be consistent whenever the law of large numbers can be invoked to give consistency of the sample moments, regardless of whether the data are actually Gaussian. This makes it an appealing foundation for developing an approach that accommodates partially observed cases.

However, inference based on the Gaussian distribution may be misleading if the true distribution is far from Gaussian. In the complete data setting, traditional maximum likelihood (ML) techniques such as likelihood ratio testing and Wald-type inference are seldom performed, with approaches based on Fisher's (Fisher, 1915) variance stabilizing transformation being much more common. An approach to estimation and inference for the correlation parameter in the setting of partially observed data based on Fisher's transformation has been developed (Li et al., 2005). All these approaches may produce misleading results if the data are strongly non-Gaussian, or if the conditions under which Fisher's transformation actually stabilizes the variance do not hold.

The approach we implement here is fully model-based and does not rely on variance stabilizing transformations. Instead, we use standard maximum-likelihood approaches for estimation and inference. The model-based framework discussed here can be used with any model for bivariate data that includes an explicit correlation parameter (including any "elliptic distribution" with density of the form $\left.c \cdot \phi\left((z-\mu)^{\prime} \Sigma^{-1}(z-\mu)\right)\right)$. Most such models will involve additional nuisance parameters describing the marginal distributions. Likelihood ratio testing and profile likelihood analysis are especially useful in settings where several nuisance parameters must be estimated along with the parameter of interest.

To explore the sensitivity of the estimates and inferences to the model specification, we also implemented our approach for the setting where the data follow a bivariate Student-t distribution. The density for this distribution when $x$ and $y$ have zero mean, unit variance and degree of freedom $d$ is

$$
f_{0}(x, y ; d)=\frac{1}{2 \pi \sqrt{1-\rho_{X Y}^{2}}}\left(1+\frac{x^{2}+y^{2}-2 \rho_{X Y} x y}{d\left(1-\rho_{X Y}^{2}\right)}\right)^{(d / 2+1)} .
$$

Note that the parameter $\rho_{X Y}$ retains the "product moment" interpretation of the Pearson correlation coefficient - that is, $\rho_{X Y}=E[x y] /(\operatorname{SD}(x) \cdot \operatorname{SD}(y))$.

\section{Partially observed data}

Our main goal here is to accommodate partially observed data. In the presence of left-, right-, and/or interval-censored data, the univariate likelihood function can be written in terms of density functions (for exact observed values), cumulative distribution functions $\left(\mathrm{CDF}, F_{\theta}(x)=\mathbb{P}(X \leq x)\right.$, for left- 
censored values), survival functions $\left(S_{\theta}(x)=\mathbb{P}(X>x)\right.$, for right-censored values) and differences between cumulative distribution functions $\left(F_{\theta}(b)-F_{\theta}(a)\right.$, for interval-censored values in an interval $(a, b])$. Here $\theta$ represents a generic vector of parameters including the correlation coefficient of interest along with any nuisance parameters.

We adopt a convention to treat all three censoring types and exact observed values in a unified representation, with all being special cases of interval censoring. Specifically, every case is known to lie in an interval $\left(x_{i}^{\text {lower }}, x_{i}^{\text {upper }}\right]$, with $-\infty<x_{i}^{\text {lower }}=x_{i}=x_{i}^{\text {upper }}<\infty$ for exact observed cases, $-\infty=x_{i}^{\text {lower }}<x_{i}^{\text {upper }}<\infty$ for left censored cases, $-\infty<x_{i}^{\text {lower }}<x_{i}^{\text {upper }}<\infty$ for interval censored cases, and $-\infty<x_{i}^{\text {lower }}<x_{i}^{\text {upper }}=\infty$ for right censored cases. In addition, we define $-\infty=x_{i}^{\text {lower }}<$ $x_{i}^{u p p e r}=\infty$ for missing cases; in this case the likelihood contribution is $F(\infty)-F(-\infty)=1-0=1$, equivalent to omitting the missing values. However, this convention will be useful to handle missing $x$ or $y$ in the bivariate case. Similar unified representations can be also found in Allison (2010) and Giolo (2004). For example, for $k_{1}$ left-censored values with LODs $x_{i}^{\text {upper }}, i=1, \ldots, k_{1}$; followed by $k_{2}-k_{1}$ interval-censored values with censoring interval bounds $\left(x_{i}^{\text {lower }}, x_{i}^{\text {upper }}\right], i=k_{1}+1, \ldots, k_{2} ; k_{3}-k_{2}$ rightcensored at values $x_{i}^{\text {lower }}, i=k_{2}+1, \ldots, k_{3}$; and $n-k_{3}$ exact values $x_{i}, i=k_{3}+1, \ldots, n$, the univariate likelihood function would be

$$
L(\theta)=\prod_{i=1}^{k_{1}} F_{\theta}\left(x_{i}^{\text {upper }}\right) \cdot \prod_{i=k_{1}+1}^{k_{2}}\left[F_{\theta}\left(x_{i}^{\text {upper }}\right)-F_{\theta}\left(x_{i}^{\text {lower }}\right)\right] \cdot \prod_{i=k_{2}+1}^{k_{3}}\left[1-F_{\theta}\left(x_{i}^{\text {lower }}\right)\right] \cdot \prod_{i=k_{3}+1}^{n} f_{\theta}\left(x_{i}\right) .
$$

For the bivariate setting of $(X, Y)$, extending the likelihood function to accommodate censored data is complicated by the number of types of data pairs to consider. Each of the $X$ and $Y$ variables can be one of the following cases: completely observed, left censored, interval censored, right censored and missing. This yields $5^{2}=25$ types of data pairs. For example, when there are only left-censored and complete data, four cases must be considered: (1) $x$ and $y$ both complete, (2) $x$ left-censored and $y$ complete, (3) $x$ complete and $y$ left-censored, and (4) both $x$ and $y$ left-censored. Each factor in the likelihood (4) is one of these four cases. For case 1, the factor is $f\left(x_{i}, y_{i}\right)$, as in the complete data situation. For case 2, the factor is $F_{X \mid Y}\left(x_{i} \mid y_{i}\right) \cdot f\left(y_{i}\right)$, where $F_{X \mid Y}\left(x \mid y_{i}\right)$ is the conditional distribution function of $X$, given $y_{i}$. For case 3 , the factor is $F_{Y \mid X}\left(y_{i} \mid x_{i}\right) \cdot f\left(x_{i}\right)$, where $F_{Y \mid X}\left(y \mid x_{i}\right)$ is the conditional distribution function of $Y$, given $x_{i}$. For case 4 , the factor is $F\left(x_{i}, y_{i}\right)=P\left(X \leq x_{i}, Y \leq y_{i}\right)$, i.e., the bivariate cumulative distribution function evaluated at $\left(x_{i}, y_{i}\right)$.

In the Gaussian model, for cases 2 and 3 we can take advantage of the fact that the conditional distributions are also Gaussian, with means and variances that are easily calculated (Rao, 1973). Thus we calculate $F_{X \mid Y}\left(x_{i} \mid y_{i}\right) \cdot f\left(y_{i}\right)$ rather than $\mathbb{P}\left(X \leq x_{i}, Y=y_{i}\right)$, because the former eases the calculation by reducing the dimension of the distribution functions from bivariate to univariate. This method can be applied to right-censored data in a similar way. In case 4 , because $F\left(x_{i}, y_{i}\right)$ does not have a closed form expression, it must be evaluated by integration of the joint density over a 2-dimensional region in the bivariate domain. Efficient methods for this calculation are provided by the mvtnorm $\mathrm{R}$ package.

Besides censoring, missing data can also be present in bivariate data (Little and Rubin, 2002). When both members of a pair are missing, the likelihood contribution factor is 1, and these observations can be omitted from the analysis. When one member of a pair is missing, the observed member of the pair can be used to improve estimation of parameters describing its marginal distribution.

The likelihood function $L(\theta)$ is the product of $n$ factors, one per observation pair, with the form of each term depending on whether members of the pair are observed, censored or missing. Table 1 lists all the possible cases of such pairs and the corresponding factor of the likelihood function in the bivariate normal case. We use the conditional distribution whenever possible to make the calculations easier.

\section{Alternative models}

When data arise from an underlying heavy tailed bivariate distribution, estimates based on the bivariate normal model may yield confidence intervals with poor coverage probabilities. This is especially a concern when partially observed data are present. We provide the bivariate Student- $t$ model as an alternative approach to estimation in this setting.

The bivariate $t$ distribution does not have the useful property held by the bivariate normal distribution that the conditional distributions have the same distributional form as the marginal distributions. Consequently, we do not have a simple expression for the conditional distributions of the bivariate $t$ distribution. Instead, we construct the likelihood through numerical integration over certain regions of the bivariate domain for each likelihood factor. Specifically, the likelihood factors 


\begin{tabular}{|c|c|c|c|}
\hline Pair & & & \\
\hline $\begin{array}{l}X o ., Y o . \\
X o ., Y r c . \\
X o ., Y m . \\
X l c ., Y l c . \\
X l c ., Y i c . \\
X r c ., Y o . \\
X r c ., Y r c . \\
X r c ., Y m . \\
X i c ., Y l c . \\
X i c ., Y i c . \\
X m ., Y o . \\
X m ., Y r c . \\
X m ., Y m .\end{array}$ & $\begin{array}{c}f\left(x_{i}, y_{i}\right) \\
{\left[1-F_{Y \mid X}\left(y_{i}^{l} \mid x_{i}\right)\right] * f_{X}\left(x_{i}\right)} \\
f_{X}\left(x_{i}\right) \\
F\left(x_{i}^{u}, y_{i}^{u}\right) \\
\mathbb{P}\left(X \leq x_{i}^{u}, y_{i}^{l}<Y \leq y_{i}^{u}\right) \\
{\left[1-F_{X \mid Y}\left(x_{i}^{l} \mid y_{i}\right)\right] * f_{Y}\left(y_{i}\right)} \\
\mathbb{P}\left(X>x_{i}^{l}, Y>y_{i}^{l}\right) \\
1-F_{X}\left(x_{i}^{l}\right) \\
\mathbb{P}\left(x_{i}^{l}<X \leq x_{i}^{u}, Y \leq y_{i}^{u}\right) \\
\mathbb{P}\left(x_{i}^{l}<X \leq x_{i}^{u}, y_{i}^{l}<Y \leq y_{i}^{u}\right) \\
f_{Y}\left(y_{i}\right) \\
1-F_{Y}\left(y_{i}^{l}\right) \\
1\end{array}$ & $\begin{array}{l}X o ., Y l c . \\
X o ., Y i c . \\
X l c ., Y o . \\
X l c ., Y r c . \\
X l c ., Y m . \\
X r c ., Y l c . \\
X \text { rc., Yic. } \\
\text { Xic., Yo. } \\
X i c ., Y r c . \\
X i c ., Y m . \\
X m ., Y l c . \\
X m ., Y i c .\end{array}$ & $\begin{array}{c}F_{Y \mid X}\left(y_{i}^{u} \mid x_{i}\right) * f_{X}\left(x_{i}\right) \\
{\left[F_{Y \mid X}\left(y_{i}^{u} \mid x_{i}\right)-F_{Y \mid X}\left(y_{i}^{l} \mid x_{i}\right)\right] * f_{X}\left(x_{i}\right)} \\
F_{X \mid Y}\left(x_{i}^{u} \mid y_{i}\right) * f_{Y}\left(y_{i}\right) \\
\mathbb{P}\left(X \leq x_{i}^{u}, Y>y_{i}^{l}\right) \\
F_{X}\left(x_{i}^{u}\right) \\
\mathbb{P}\left(X>x_{i}^{l}, Y \leq y_{i}^{u}\right) \\
\mathbb{P}\left(X>x_{i}^{l}, y_{i}^{l}<Y \leq y_{i}^{u}\right) \\
{\left[F_{X \mid Y}\left(x_{i}^{u} \mid y_{i}\right)-F_{X \mid Y}\left(x_{i}^{l} \mid y_{i}\right)\right] * f_{Y}\left(y_{i}\right)} \\
\mathbb{P}\left(x_{i}^{l}<X \leq x_{i}^{u}, Y>y_{i}^{l}\right) \\
F_{X}\left(x_{i}^{u}\right)-F_{X}\left(x_{i}^{l}\right) \\
F_{Y}\left(y_{i}^{u}\right) \\
F_{Y}\left(y_{i}^{u}\right)-F_{Y}\left(y_{i}^{l}\right)\end{array}$ \\
\hline
\end{tabular}

Table 1: Contributing factors to the likelihood function for each case of observed, censored or missing values in pairs from the bivariate normal distribution. "o." = observed; "lc." = left censored; "rc." =right censored; "ic." = interval censored; "m." = missing. $x_{i}^{l}=x_{i}^{\text {lower }} ; x_{i}^{u}=x_{i}^{\text {upper }} ; y_{i}^{l}=y_{i}^{\text {lower }} ; y_{i}^{u}=y_{i}^{\text {upper }}$.

are given by

$$
\int_{x_{i}^{\text {lower }}}^{x_{i}^{\text {upper }}} \int_{y_{i}^{\text {lower }}}^{y_{i}^{\text {upper }}} f(\mu, v) d \mu d v,
$$

where $f(x, y)$ is the bivariate $t$ density, and $\left(x_{i}^{\text {lower }}, x_{i}^{\text {upper }}\right)$ are the censoring intervals as defined in Section 24.1.2. Table 2 lists the likelihood contribution for each case. We use the mvtnorm package in $\mathrm{R}$ to approximate these values.

\begin{tabular}{|c|c|c|c|}
\hline Pair type & Factor to the likelihood & Pair type & Factor to the likelihood \\
\hline X o., Yo. & $f\left(x_{i}, y_{i}\right)$ & X o., Y lc. & $\int_{-\infty}^{y_{i}^{u}} f\left(x_{i}, v\right) d v$ \\
\hline$X o ., Y r c$. & $\int_{y_{i}^{l}}^{\infty} f\left(x_{i}, v\right) d v$ & $X o ., Y$ ic. & $\int_{u^{l}}^{y_{i}^{u}} f\left(x_{i}, v\right) d v$ \\
\hline Xo., Y m. & $f_{X}\left(x_{i}\right)$ & $X l_{c .}, Y_{o}$ & $\int_{-\infty}^{x_{i}^{u}} f\left(\mu, y_{i}\right) d \mu$ \\
\hline$X l c ., Y l c$. & $\int_{-\infty}^{y_{i}^{u}} \int_{-\infty}^{x_{i}^{u}} f(\mu, v) d \mu d v$ & $X l c ., Y r c$. & $\int_{y_{i}^{l}}^{\infty} \int_{-\infty}^{x_{i}^{u}} f(\mu, v) d \mu d v$ \\
\hline X lc., Y ic. & $\int_{y_{i}^{l}}^{y_{i}^{u}} \int_{-\infty}^{x_{i}^{u}} f(\mu, v) d \mu d v$ & $X l c ., Y m$. & $F_{X}\left(x_{i}^{u}\right)$ \\
\hline Xrc., Yo. & $\int_{x_{i}^{l}}^{\infty} f\left(\mu, y_{i}\right) d \mu$ & $X r c ., Y l c$ & $\int_{-\infty}^{y_{i}^{u}} \int_{x_{i}^{l}}^{\infty} f(\mu, v) d \mu d v$ \\
\hline$X r c ., Y r c$. & $\int_{y_{i}^{l}}^{\infty} \int_{x_{i}^{u}}^{\infty} f(\mu, v) d \mu d v$ & $X r c ., Y i c$ & $\int_{y_{i}^{l}}^{y_{i}^{u}} \int_{x_{i}^{l}}^{\infty} f(\mu, v) d \mu d v$ \\
\hline$X r c ., Y m$. & $1-F_{X}\left(x_{i}^{l}\right)$ & Xic., Yo. & $\int_{x_{i}^{l}}^{x_{i}^{u}} f\left(\mu, y_{i}\right) d \mu$ \\
\hline Xic., Y lc. & $\int_{-\infty}^{y_{i}^{u}} \int_{x_{i}^{l}}^{x_{i}^{u}} f(\mu, v) d \mu d v$ & Xic., Y rc. & $\int_{y_{i}^{l}}^{\infty} \int_{x_{i}^{l}}^{x_{i}^{u}} f(\mu, v) d \mu d v$ \\
\hline$X i c ., Y i c$. & $\int_{y_{i}^{l}}^{y_{i}^{u}} \int_{x_{i}^{l}}^{x_{i}^{u}} f(\mu, v) d \mu d v$ & $X i c ., Y m$. & $F_{X}\left(x_{i}^{u}\right)-F_{X}\left(x_{i}^{l}\right)$ \\
\hline $\begin{array}{l}X m ., Y o . \\
X m ., Y r c . \\
X m ., Y m .\end{array}$ & $\begin{array}{c}f_{Y}\left(y_{i}\right) \\
1-F_{Y}\left(y_{i}^{l}\right) \\
1\end{array}$ & $\begin{array}{l}X m ., Y l c . \\
X m ., Y i c .\end{array}$ & $\begin{array}{c}F_{Y}\left(y_{i}^{u}\right) \\
F_{Y}\left(y_{i}^{u}\right)-F_{Y}\left(y_{i}^{l}\right)\end{array}$ \\
\hline
\end{tabular}

Table 2: Contributing factors to the likelihood function for each case of observed, censored or missing values in pairs from the bivariate $t$-distribution. "o." = observed; "lc." = left censored; "rc." =right censored; "ic." = interval censored; "m." = missing. $x_{i}^{l}=x_{i}^{\text {lower }} ; x_{i}^{u}=x_{i}^{\text {upper }} ; y_{i}^{l}=y_{i}^{\text {lower }} ; y_{i}^{u}=y_{i}^{\text {upper }}$.

\section{Inference}

For large $n$, the likelihood ratio (LR) test for the null hypothesis $\rho_{X Y}=\rho_{0}$ is obtained by evaluating the log profile likelihood function at the maximum likelihood estimator (MLE) and at a given value 
$\rho_{0}$, taking twice the (positive) difference between them, and comparing to a chi-squared distribution with 1 degree of freedom (Murphy and van der Vaart, 2000). The $p$-value for the test is the chi-squared probability of a value as or more extreme as the one obtained. A profile likelihood-based confidence interval for $\rho_{X Y}$ is obtained by inverting the likelihood ratio test, i.e., by finding the values $\left(\rho_{L}, \rho_{U}\right)$ above and below the profile MLE for which the LR test would first reject (at significance level $\alpha$ ) the null hypothesis that $\rho_{X Y}=\rho_{L}$, and similarly $\rho_{U}$. Specifically, the confidence interval with coverage probability $1-\alpha$ is constructed to be the set

$$
\left\{\rho:-2\left[\ell(\rho)-\max _{\rho} \ell(\rho)\right]<q_{\chi_{1}^{2}}(1-\alpha)\right\}
$$

where $\ell(\rho)=\max _{\mu_{X}, \mu_{Y}, \sigma_{X}, \sigma_{Y}} \ell\left(\mu_{X}, \mu_{Y}, \sigma_{X}, \sigma_{Y}, \rho\right)$ is the profile log likelihood for $\rho$ and $q_{\chi_{1}^{2}}(1-\alpha)$ is the $1-\alpha$ upper critical value of the chi-squared distribution with 1 degree of freedom (Venzon and Moolgavkar, 1988; Stryhn and Christensen, 2003).

Profile likelihood based confidence intervals have some advantages over other methods such as the commonly used Wald-type confidence intervals obtained from the limiting distribution of the MLE. Wald-type intervals may give poor performance when the parameter is close to the boundary of its domain, in which case the parameter estimate assumes a skewed distribution (Stryhn and Christensen, 2003). The profile likelihood based approach is robust to this skewness by providing an asymmetric confidence interval estimate. Zhou et al. (2012) compared confidence interval estimates for a logistic regression setting using (1) the Wald method, (2) the Bootstrap method and (3) the profile likelihood based method. In our setting, the profile likelihood based method is more computationally efficient than the bootstrap method and is more robust to cases of parameter estimates with markedly skewed distributions than the Wald method.

\section{An $R$ package, clikcorr}

We implemented an R package clikcorr ( $\mathrm{Li}$ et al., 2016) to facilitate inference on the correlation coefficient from bivariate data in the presence of censored and/or missing values. The package is able to handle all combinations of observed, censored (left-, right-, or interval-censored) or missing data on each of the bivariate components. Most of the currently available software for estimating correlation coefficient between bivariate censored data focus on the left-truncated and right-censored data (Li et al., 2005; Schemper et al., 2013). To the best of our knowledge, clikcorr is the first available software that can handle data with a complex mixture types of censoring and missing. The package also has graphical functions to help visualize bivariate censored data and the shape of the profile log likelihood function.

We note the large set of routines for left-censored data given in the NADA (Non-detects and Data Analysis) package (Helsel, 2012, 2005). NADA currently has no function for the Pearson correlation coefficient with left-censored data, although it does include a function for Kendall's $\tau$. The implementation described here is not currently included in NADA.

clikcorr requires a special input data format. Each input variable is represented by two columns for the interval bounds discussed in section 24.1.3, with $\pm \infty$ replaced by "NA" values. The same input data format is also adopted in the LIFEREG procedure of SAS software (Allison, 2010) and in the "Surv" class of the survival package (Therneau, 2015) in R when type=interval2. Table 3 gives an example dataset formatted for input into clikcorr, with a pair of variables, Variable1 and Variable2, each given with lower and upper interval endpoints. For the first sample ID, both variables are exactly observed at values 10.9 and 37.6 respectively. For the second to the fifth samples, Variable1 is exactly observed but Variable2 is, in order, left-censored at 7.6, right censored at 26.7, interval-censored at $(11.7,20.9)$ and missing. For sample ID 6, both variables are left censored, and for sample ID 7, both variables are interval censored. clikcorr functions rightLeftC2DF and intervalC2DF can transform input data from "Surv" class formats into the clikcorr required input format. rightLeftC2DF transforms the right- or left- censored data in the "Surv" class and "intervalC2DF" transforms interval-censored data (either interval or interval2) in the "Surv" class.

clikcorr has three main functions, one estimating function and two graphical functions. The estimating function calculates the correlation coefficient estimate, its confidence interval and the $p$ value from the likelihood ratio test of the null hypothesis that the underlying true correlation coefficient is zero. The user can specify either a bivariate normal (the default) or a bivariate $t$ distribution to use for inference.

Below is an example of calling the estimation function and the output assuming (by default) the bivariate normal distribution. Here ND is an example dataset contained in the clikcorr package. See Li et al. (2016) for details about the ND dataset. ("t1_OCDD", "t1_HxCDF_234678") and ("t2_OCDD", "t2_HxCDF_234678") are column names for the lower and upper interval bounds for input variables 


\begin{tabular}{ccccc}
\hline Sample ID & $\begin{array}{c}\text { Lower bound of } \\
\text { the 1st variable }\end{array}$ & $\begin{array}{c}\text { Upper bound of } \\
\text { the 1st variable }\end{array}$ & $\begin{array}{c}\text { Lower bound of } \\
\text { the 2nd variable }\end{array}$ & $\begin{array}{c}\text { Upper bound of } \\
\text { the 2nd variable }\end{array}$ \\
\hline 1 & 10.9 & 10.9 & 37.6 & 37.6 \\
2 & 12.8 & 12.8 & $\mathrm{NA}$ & 7.6 \\
3 & 8.7 & 8.7 & 26.7 & $\mathrm{NA}$ \\
4 & 13.2 & 13.2 & 11.7 & 20.9 \\
5 & 9.8 & 9.8 & $\mathrm{NA}$ & $\mathrm{NA}$ \\
6 & $\mathrm{NA}$ & 10.0 & $\mathrm{NA}$ & 6.9 \\
7 & 11.4 & 16.3 & 10.8 & 18.7 \\
\hline
\end{tabular}

Table 3: An example input data frame for clikcorr with two input variables.

"OCDD" and "HxCDF_234678", respectively . $\mathrm{cp}$ is the user specified coverage probability (confidence level) of the confidence interval, with the default set to 0.95 . The print method of "clikcorr" class outputs the coefficients matrix, which contains the estimated correlation coefficient coefficients, the lower and upper bounds for the confidence interval CI. lower and CI.upper, and the $p$-value, $\mathrm{p}$. value, from the likelihood ratio test of the null hypothesis that $\rho_{X Y}=0$.

$\mathrm{R}>\operatorname{data}(\mathrm{ND})$

$\mathrm{R}>\log N \mathrm{R}<-\log (\mathrm{ND})$

$\mathrm{R}>$ clikcorr (data $=\operatorname{logND}$, lower1 = "t1_OCDD", upper1 = "t2_OCDD",

+ lower2 = "t1_HxCDF_234678", upper2 = "t2_HxCDF_234678", cp=.95)

Call:

clikcorr.default (data $=\operatorname{logND}$, lower1 = "t1_OCDD", upper1 = "t2_OCDD",

lower2 = "t1_HxCDF_234678", upper2 = "t2_HxCDF_234678", сp $=0.95$ )

coefficients $95 \% \mathrm{CI}$.lower $95 \%$ CI.upper p.value

$0.472657898-0.006538307 \quad 0.7696201790 .053083585$

The summary method further outputs the vector of estimated means \$Mean, the estimated variancecovariance matrix \$Cov and the log likelihood value at the MLE \$Loglike.

R> obj <- clikcorr (data=logND, lower1="t1_OCDD", upper1="t2_OCDD",

+ lower2="t1_HxCDF_234678", upper2="t2_HxCDF_234678", $\mathrm{cp}=.95$ )

R> summary (obj)

$\$ c a l 1$

clikcorr.default (data = logND, lower1 = "t1_OCDD", upper1 = "t2_OCDD", lower2 = "t1_HxCDF_234678", upper2 = "t2_HxCDF_234678", $\mathrm{cp}=0.95$ )

\$coefficients

coefficients 95\%CI.lower 95\%CI.upper p.value

$\begin{array}{llll}0.472657898 & -0.006538307 & 0.769620179 & 0.053083585\end{array}$

\$mean

[1] $5.3706036-0.4655811$

$\$ \operatorname{Cov}$

$[, 1] \quad[, 2]$

$[1] \quad$,

$[2] \quad 0.3100190 \quad$,

$\$ \log l i k$

[1] -37.97112

$\operatorname{attr}($, "class")

[1] "summary.clikcorr"

As we will illustrate in a later section, when data are generated from a heavy tailed distribution, inference assuming the bivariate normal distribution can result in poor performance. The option "dist=t" can be used to specify a bivariate $t$ distribution. The option "df" specifies the degrees of freedom (d.f.) of the bivariate $t$ distribution and by default is set to 4 . Smaller d.f. give $t$ distributions 
with heavier tails; larger d.f. (e.g., $>30$ ) give $t$ distributions that are similar to the normal distribution. Below is an example of calling the estimation function using dataset ND, and the output assuming the bivariate $t$ distribution. The variable names are the same as given above.

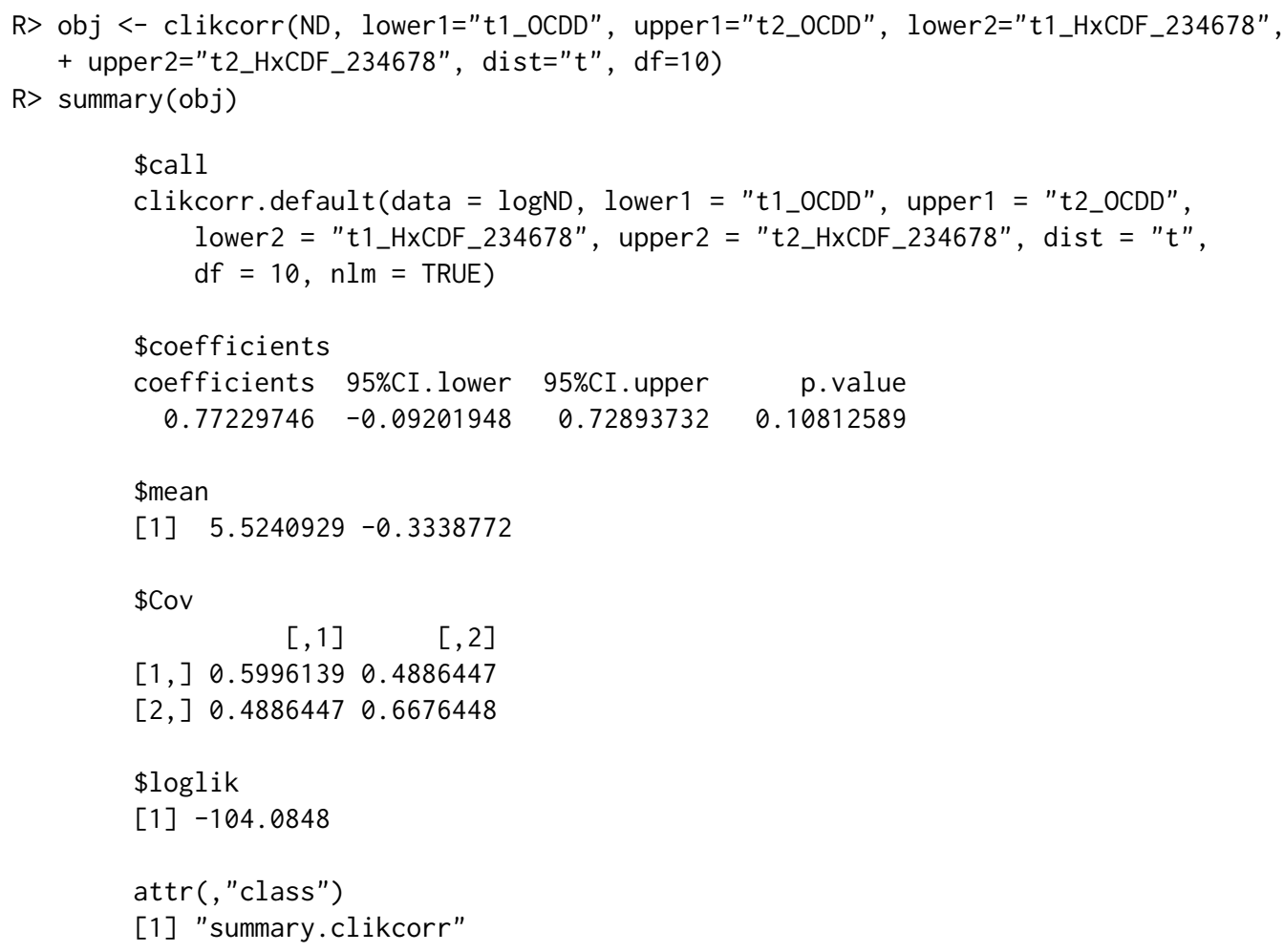

clikcorr also provides two graphical functions. Visualizing the data and the analytic outputs is often useful, and is sometimes invaluable for understanding the results. A barrier to graphing censored data is finding a way to plot the range of possible data values. The function splot provides an effective way of visualizing bivariate censored and/or missing data. With splot, censored values can be identified with different colors or plotting symbols, depending on the data types, as described in sections 24.1.2 and 24.1.3. For example, the plotting symbol could be an arrow showing the direction of uncertainty. Since the ND dataset in clikcorr package only contains right censored data points, to demonstrate the fact that clikcorr can provide a visual representation for all types of censored and/or missing data, in the following examples, we illustrate the clikcorr plotting functions using a simulated dataset. Denote by SimDat an input data matrix, generated from a tri-variate normal distribution with pairwise correlation coefficient 0.5 and with missing values and all types of censoring randomly introduced. Let "L1" and "U1" be column names for the lower and upper interval bounds for input variable 1. Similar notations are also adopted for variable 2 and variable 3 . A single scatterplot of Variable 2 versus Variable 1, or ("L2", "U2") versus ("L1", "U1"), would be plotted by calling splot2 (SimDat, c("L1", "L2"), c("U1", "U2"). The following syntax calls splot and produces a scatterplot matrix with all combinations of three input variables, as shown in Figure 1. Note that a censored observation has its position indicated by the tail of an arrow and the head of the arrow is pointed to the censoring direction.

R> splot(SimDat, c("L1", "L2", "L3"), c("U1", "U2", "U3"))

The S3 function plot of the clikcorr class produces a plot of the profile log likelihood function. The following syntax calls the plot function using the bivariate normal and the bivariate $t$-distributions respectively. The output profile plots are given in Figure 2, and illustrate that specifying the appropriate distribution is important for both optimum point and confidence interval estimation.

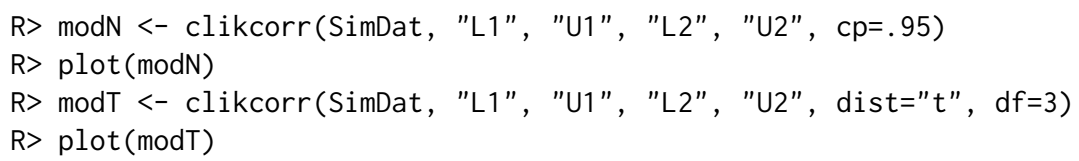

The clikcorr main estimating function requires that the following other R functions be loaded: the R package mvtnorm (Genz and Bretz, 2009; Genz et al., 2012; Hothorn et al., 2013), as well as function "optim", and function "integrate" when using the bivariate $t$-distribution. 

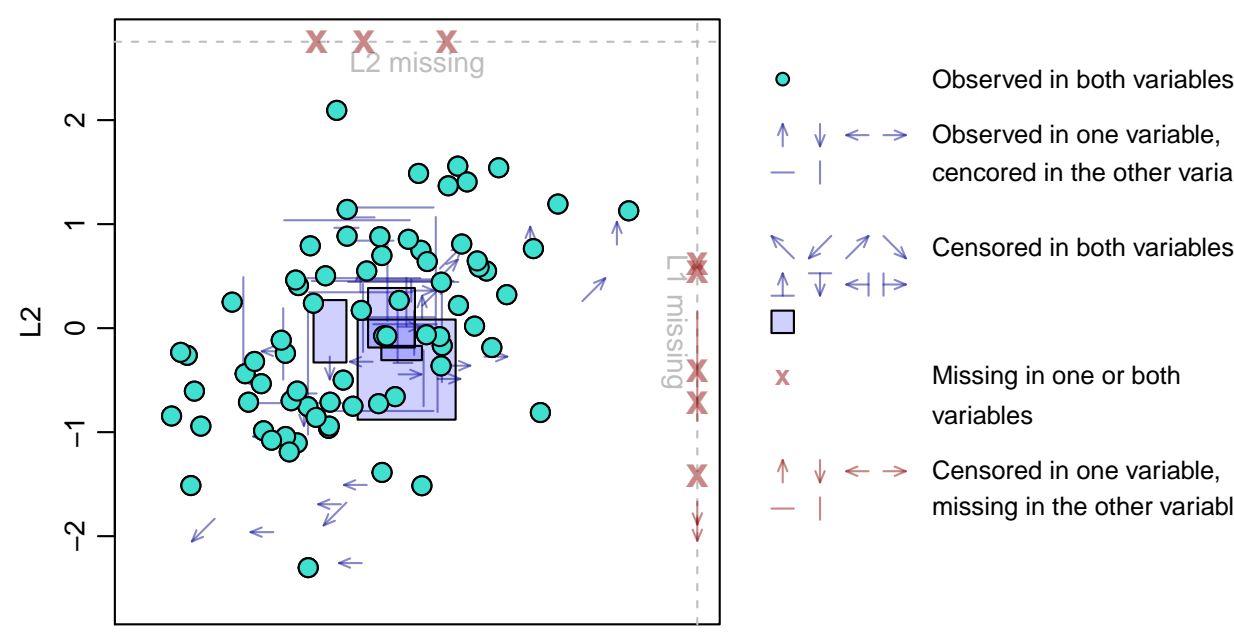

$\uparrow \downarrow<\rightarrow$ Observed in one variable,

- I cencored in the other variable

$\nwarrow \swarrow \nearrow \searrow$ Censored in both variables

^ $\downarrow<\mapsto \mapsto$

x Missing in one or both

variables

$\uparrow \downarrow \leftarrow \rightarrow$ Censored in one variable,

- 1 missing in the other variable
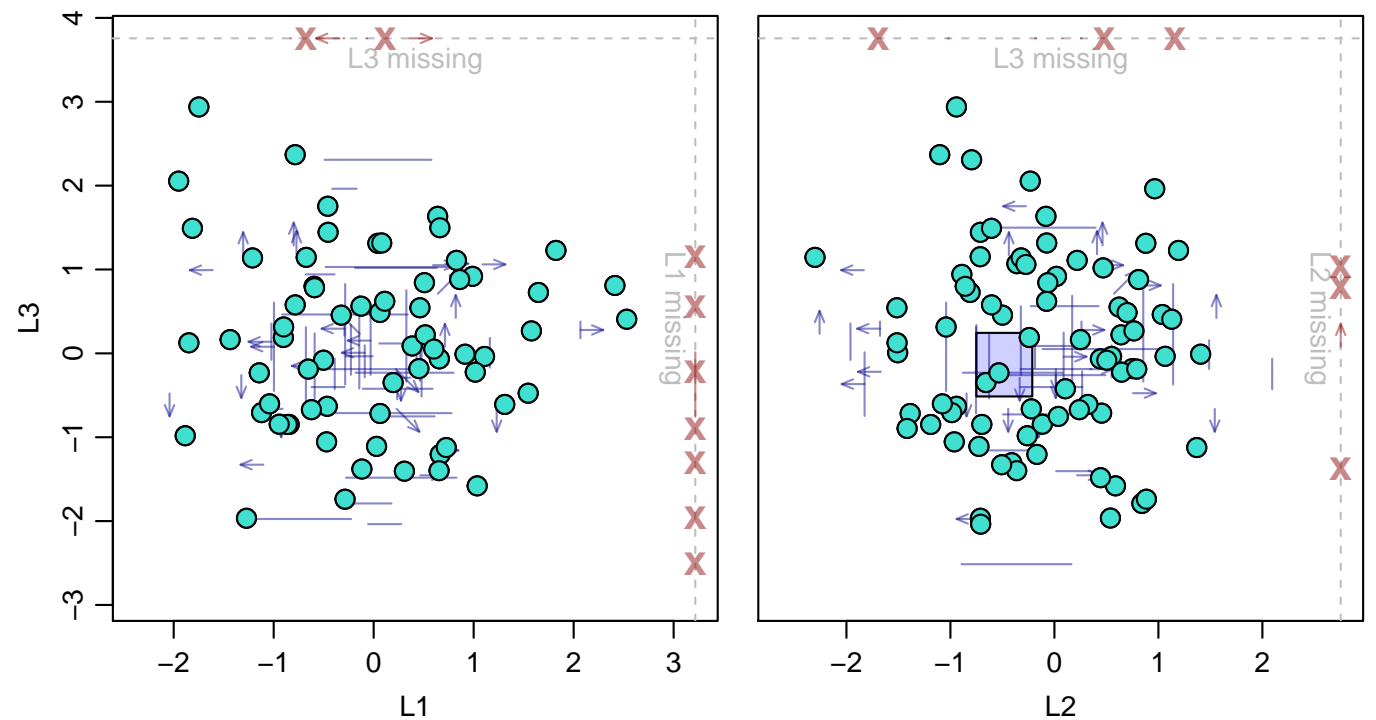

Figure 1: Example scatter plots from a simulated data frame.

\section{Simulation studies}

Extensive simulations have been done to demonstrate different aspects of the performance of clikcorr under various settings of sample size, percent censoring, and underlying distribution. We show these results in the following subsections.

\section{Coverage probability of the confidence interval}

First, we investigate the coverage probability of the confidence intervals with two types of right censoring, termed "fixed point" and "random", as described further below. Data were generated from the bivariate normal distribution, and inference assumed the same distribution.

In the fixed point right censoring setting, all observations were censored at the same value. Different fixed points were calculated to give expected censoring rates of $0 \%, 25 \%$ and $75 \%$. For the random right censoring setting, values were censored at randomly generated thresholds, which were generated from normal distributions with means set at different values to produce data with specific expected censoring proportions. Tables 4 and 5 give the simulated coverage probabilities for different settings of sample sizes and censoring rates. In the completely observed cases in Table 4, coverage probabilities calculated using the Fisher transformation rather than the profile likelihood approach are given in parentheses for comparison.

It can be seen from Tables 4 and 5 that clikcorr gives satisfying confidence interval coverage probabilities in both the fixed and random censoring settings. In the completely observed cases, coverage probabilities for both profile likelihood and Fisher transformation based confidence intervals 

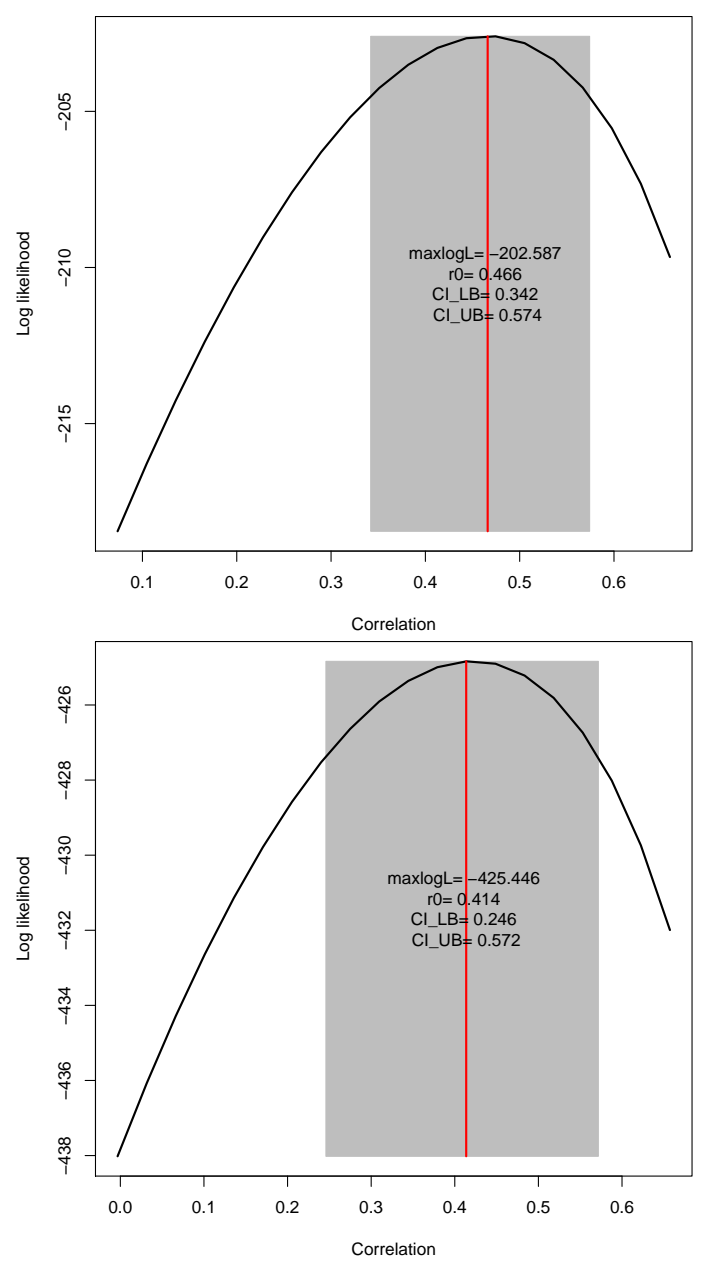

Figure 2: Example of profile likelihood plots. Input data are simulated from the bivariate normal distribution with true $\rho_{X Y}=0.5$. The upper panel shows the profile likelihood using the bivariate normal distribution; the lower panel shows the profile likelihood using the bivariate $t$-distribution.

are very close to the nominal level of 0.95

\section{Run time, bias and mean squared error (MSE)}

Table 6 shows the run time of clikcorr under different settings of sample sizes and censoring proportion configurations, using the bivariate normal distribution for both data generation and inference. The censoring proportion configurations, for example "X15\%, XY15\%, $Y 15 \%$ " means that $15 \%$ of the observations are censored only for $X, 15 \%$ are censored for both $X$ and $Y$, and $15 \%$ are censored only for $Y$. It can be seen that longer run time is needed for larger sample size, larger proportion with both $X$ and $Y$ being censored, or larger magnitude of the correlation. A typical run time ranges from a few seconds to a few minutes.

For the bivariate $t$ distributions, the run time is generally slower than for the bivariate normal distributions in the same setting. The reason for this is that the bivariate $t$ setting requires numerical integration to calculate the joint distribution over a 2-dimensional domain as illustrated in (5) in the case of either $X$ or $Y$ censored, whereas the analogous calculation for the bivariate normal setting has a closed form.

Table 7 shows the estimates of the bias and MSE for inference on $\rho$ using the bivariate normal distribution for data generation and inference, and with random right censoring. Essentially, clikcorr provides an unbiased estimator of the correlation coefficient. 


\section{Gaussian versus Student $t$ models}

When the underlying marginal distributions are heavy tailed rather than normal, basing inference on the bivariate normal can result in poor confidence interval coverage probabilities. This is illustrated in Table 8, where the data were generated from correlated marginal $t$ distributions and the correlation coefficients were estimated using the bivariate normal distribution. When the degrees of freedom of the underlying marginal $t$ distributions are small ( $\sim 3$ to 5$)$ or the true underlying correlation coefficient is very high $(\sim 0.9)$, the confidence intervals have poor coverage probabilities $(\sim 60 \%$ to $80 \%)$.

The coverage probabilities within the parentheses in Table 8 are for confidence intervals constructed using the bivariate $t$ distributions. Compared to inference based on the bivariate normal distribution, using the bivariate $t$ distribution provides much better coverage probabilities in settings with very heavy-tailed distributions and high correlations.

The clikcorr package does not provide a way to test whether the data are drawn from a heavy tailed distribution or not, nor does it estimate the best value of d.f. to use. The users could refer to Bryson (1974); Resnick (2007) or use QQ plots to guide their decision on which model to use.

\section{Dioxin exposure datasets}

In this section, we used clikcorr to analyze two real world datasets and compared the results to conventional approaches.

\section{Owl feather data}

The first dataset was analyzed in (Jaspers et al., 2013) which used an early version of clikcorr to estimate correlations for left-censored (below detection) data. The data contain Perfluorooctane sulfonate (PFOS) substances in feathers and soft tissues (liver, muscle, adipose tissue and preen glands) of barn owl road-kill victims collected in the province of Antwerp, Belgium. Figure 3 gives an example scatter plot (upper panel) and an example plot of the profile log likelihood of the correlation between PFOS in liver tissue and feathers using clikcorr. Figure 4 shows the scatter plot matrix across tail feather and the tissues generated by clikcorr. The asymmetrical shape of the profile likelihood reflects the asymmetrical sampling distribution, and leads to a confidence interval that is longer to the left than to the right of the point estimate.

\section{NHANES data}

In the second analysis using clikcorr, we analyzed dioxin data from the 2002-2003 National Health and Nutrition Examination Survey (NHANES). NHANES is one of the most widely-used datasets describing the health and nutritional status of people residing in the US. The data we analyzed contained 22 chemicals, including 7 dioxins, 9 furans and 6 polychlorinated biphenyls (PCBs) across 1643 individuals. Correlations between these chemicals are common, and are of interest for several reasons. First, dioxins with high correlations measured in the environment may arise from the same or similar sources. In addition, levels of two chemicals in the body that have high correlations may have similar pathways for entering the body. For two chemicals with high correlations, it may be sufficient to monitor one of them (to reduce cost), as was the goal in Jaspers' barn owl example. Finally, exposure to pairs of correlated chemicals may confound an investigation of health effects, since distinguishing the individual effects of each chemical would be difficult. Therefore estimating correlations between those compounds is of scientific interest. The chemicals in our dioxin data are all subject to detection limits, and have a wide range of percentage of left-censored values. It is important to incorporate information from partially observed cases in the data to provide accurate estimation and inference results on the correlations between the compounds.

The pairwise correlation coefficients calculated from clikcorr are given in upper-right triangle of Table 9. For each pair, the MLE of the correlation coefficient and associated LRT $p$-value (in parentheses) are calculated assuming the bivariate normal distribution. The highly correlated pairs (with estimated correlation coefficient $\geq .80$ ) are in boldface type. Chemicals within each group (dioxin, furan or PCB) tend to be more highly correlated than chemicals from different groups. Abbreviated names of each chemical were used in Table 9. The full names and the detail information about each chemical can be found at Agency for Toxic Substances and Disease Registry (ATSDR) (http://www. atsdr.cdc.gov/substances), which is part of the U.S. Centers for Disease Control and Prevention. The chemical indices and names are corresponding as following: for dioxind, $\mathrm{d} 1=T C D D ; \mathrm{d} 2=P e C D D ; \mathrm{d} 3=H x C D D 123478 ; \mathrm{d} 4=H x C D D 123678 ; \mathrm{d} 5=H x C D D 123789$; 

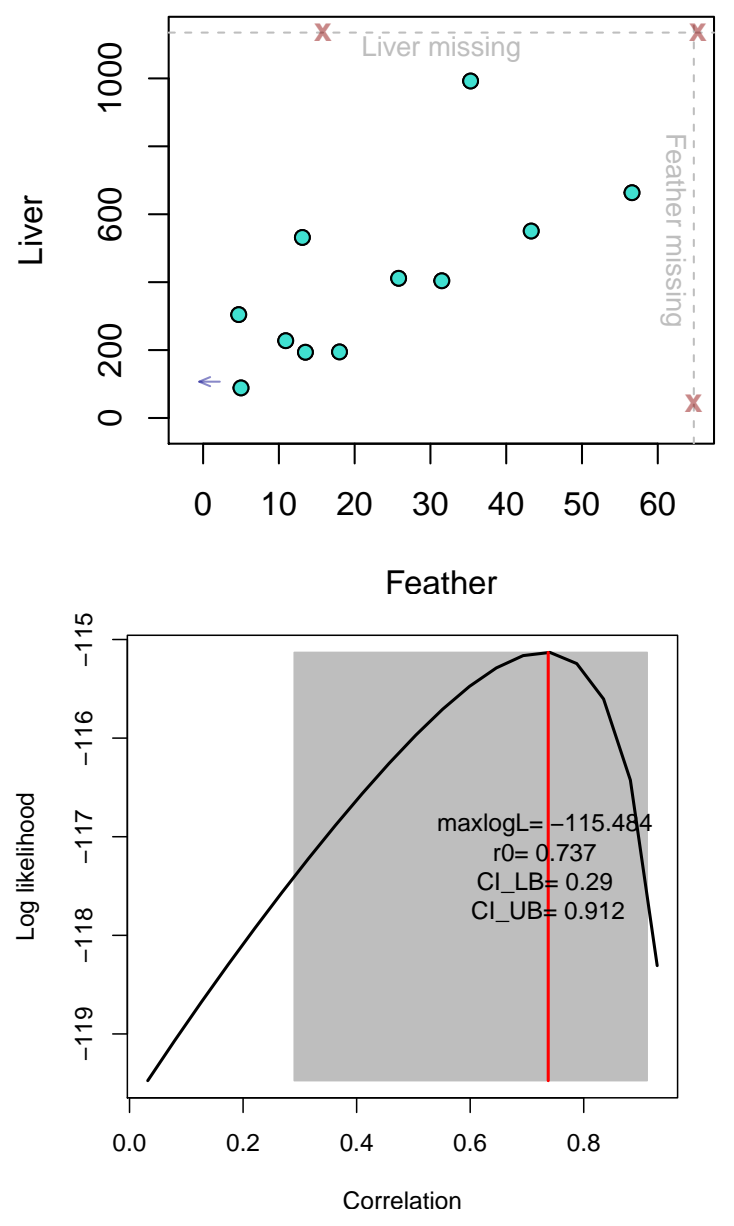

Figure 3: Output graphics from analyzing the owl feather data using clikcorr. Upper panel: scatter plot of PFOS in liver tissue vs. feathers; Lower panel: profile log likelihood plot for the correlation coefficient between PFOS concentration in liver and in feathers.

$\mathrm{d} 6=H p C D D ; \mathrm{d} 7=O C D D$. For furan, $\mathrm{f} 1=T C D F ; \mathrm{f} 2=P e C D F 12378 ; \mathrm{f3}=P e C D F 23478 ; \mathrm{f} 4=H x C D F 123478$; $\mathrm{f} 5=H x C D F 123678 ; \mathrm{f6}=H x C D F 234678 ; \mathrm{f} 7=H p C D F 1234678 ; \mathrm{f} 8=H p C D F 1234789 ; \mathrm{f} 9=O C D F$. For PCB, $\mathrm{p} 1=$ PCB105; $2=P C B 118 ; \mathrm{p} 3=P C B 156 ; \mathrm{p} 4=P C B 157 ; \mathrm{p} 5=P C B 167 ; \mathrm{p} 6=P C B 189 . \quad$-values less than 0.01 are recoded as 0 's in Table 9.

The pairs of chemicals with the highest correlation in each group are shown in Figure 6. Both the MLE of the correlation coefficient calculated from clikcorr $\hat{\rho}_{\text {clikcorr }}$, and the Pearson correlation coefficients calculated from only the complete cases $\hat{\rho}_{\text {complete, }}$ are given, along with the complete case sample size, $n_{\text {complete }}$. When the proportion of pairs with censored values is low, $\hat{\rho}_{\text {clikcorr }}$ and $\hat{\rho}_{\text {complete }}$ are very similar; on the other hand, when the proportion of pairs with censored values (in either or both variables) is high, the two correlation coefficients are different since $\hat{\rho}_{\text {clikcorr }}$ can utilize the extra information from the censored data.

Some chemicals in the dataset appear to have highly skewed marginal distributions. It is common to apply a symmetrization transformation in such a setting. The correlation coefficient between transformed values estimates a different population parameter, but this parameter may be of as much or greater inference than the correlation computed on the original scale. To demonstrate the effect of such a transformation, we log-transformed the data for each chemical and recalculated the correlation coefficient using the bivariate normal model. The estimation and inference results from the transformed data are given in the lower-left triangle in Table 9.

Using the bivariate normal model for transformed data is an example of using bivariate Gaussian copulas to allow a wide range of distributions to be analyzed using software developed for Gaussian models. A general approach of this type would be to transform $X$ using $F^{-1} \circ \hat{F}_{x}$, where $\hat{F}_{x}$ is the empirical CDF of $X$ and $F$ is the standard Gaussian CDF ( $Y$ would be transformed analogously). This approach is quiet flexible, and would allow clikcorr to be used for correlation parameter inference in a wide range of settings. One limitation of this approach is that the marginal transformations 

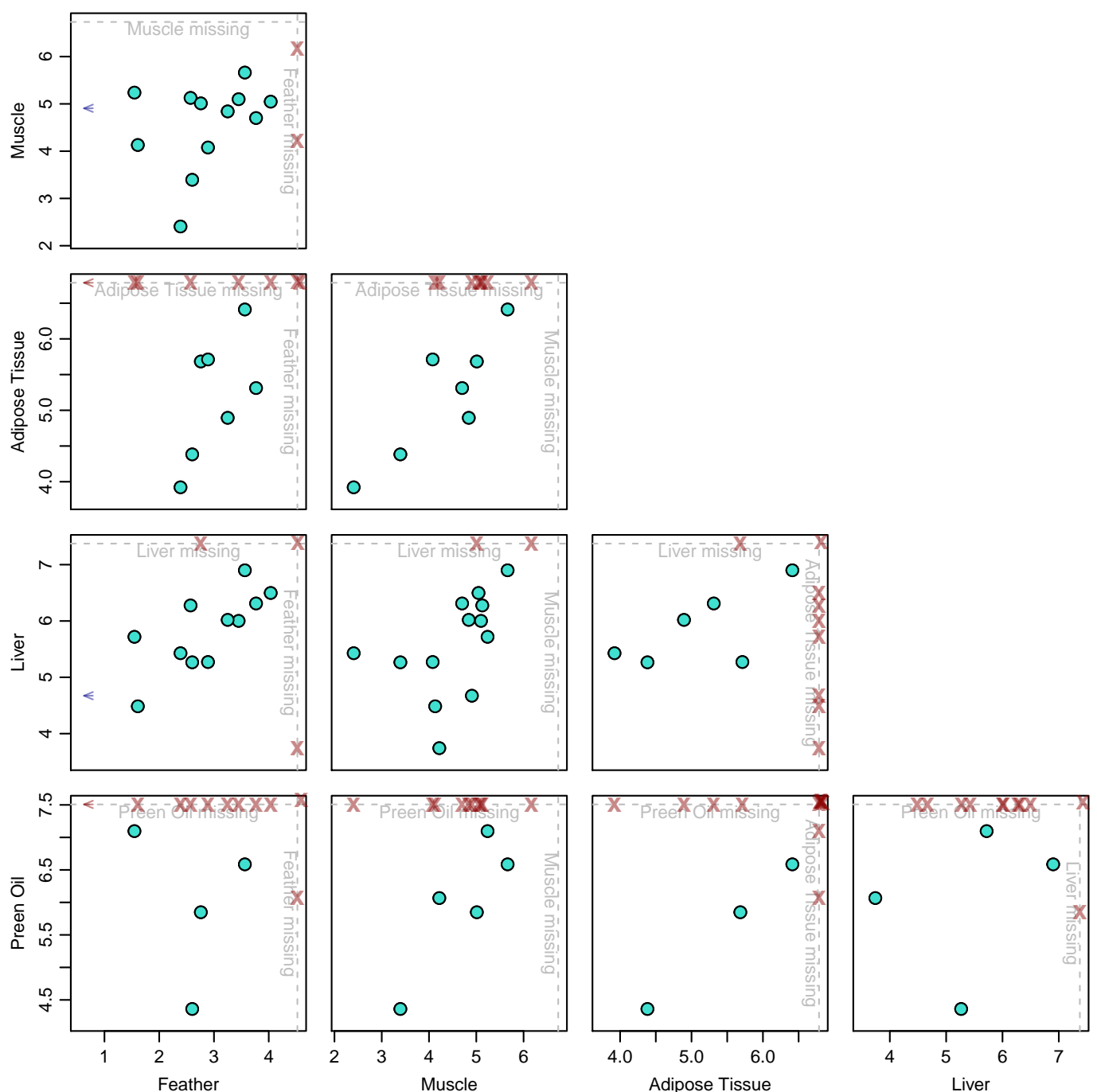

Figure 4: Scatter plot matrix from analyzing the owl feather data using clikcorr.

are estimated from the data, but the uncertainty in this estimation is not propagated through to the estimation of the correlation parameter. This would mainly be a concern with small datasets.

Figure 5 demonstrates the difference between using data on the original and the log-transformed scales. When the observed marginal distributions are highly skewed, assuming a bivariate normal true distribution could lead to false results. For example, the correlation effect could be leveraged away by the "outlier" points on tail of either marginal distributions. As in Figure 5 (c), the original values between chemicals $O C D D(\mathrm{~d} 7)$ and $H x C D F 234678$ (f6) were not significantly correlated due to the divergent effect of the points on the marginal tails. While after the log transformation, the marginal sample distributions are more normally shaped and thus make the bivariate normal assumption more likely to be true, and the two chemicals are significantly correlated with a moderate correlation coefficient.

\section{Further details on clikcorr}

This section gives technical details regarding function maximization and gives some examples of additional plotting options.

We note that clikcorr depends on the R function optim, which is used to find the maximum of the log likelihood, or profile log likelihood. For some starting values of $\mu_{X}, \mu_{Y}, \sigma_{X}, \sigma_{Y}$ and $\rho_{X Y}$, the procedure gives an error message "function cannot be evaluated at initial parameters". In such cases, manually adjusting the initial parameter values is recommended.

Figure 7 illustrates two example profile log likelihood functions for parameters of the bivariate 
distribution of PFOS in Tail feather and Adipose tissue in the owl feather dataset. Each plot shows the log likelihood for certain parameters with the other parameters fixed. In the upper panel, the $\log$ likelihood of $\left(\log \left(\sigma_{X}^{2}\right), \log \left(\sigma_{Y}^{2}\right)\right)$ remains at $-\infty$ over the lower left triangular region of the plot. So if the starting values of $\sigma_{X}$ and $\sigma_{Y}$ are set such that the pair $\left(\log \left(\sigma_{X}^{2}\right), \log \left(\sigma_{Y}^{2}\right)\right)$ falls in that region, then the value of the joint log likelihood will be trapped in the region and the above error message will show up. In the lower panel graph, the log likelihood of the covariance drops steeply when the covariance parameter value is greater than 2000. Therefore, if the starting value of $\rho_{X Y}$ is set such that the starting covariance is much larger than 2000, say 5000, then the profile log likelihood might underflow the limit of a floating number adopted in the "optim" function and give the above error message.

It is worth the effort to make the input starting parameter values as close to the true parameters as possible, if these are known. By default, clikcorr uses the sample means, the log transform of sample variances and the sample correlation based on the complete observations as the starting values. It also allows the user to specify the starting values and pass them to the "optim" function by using the option sv. This option is especially useful when the number of exact observations is small. For example, if a dataset contains only one complete observation, then the starting values of $\left(\log \left(\sigma_{X}^{2}\right), \log \left(\sigma_{Y}^{2}\right)\right)$ can not be effectively estimated (both will be estimated as $-\infty$ ). In such cases, it is suggested that users make their own reasonable guesses on the starting parameter values rather than using the default. An example is given in the following syntax:

R> clikcorr(owlData, "feather_L", "feather_U", "AT_L", "AT_U", cp=.95, dist=t, $+d f=5, s v=c(23,240,6,1300,12))$

The default optimization method for searching for the MLE in clikcorr is the default method used in "optim", which is the Nelder-Mead method (R Core Team, 2013b). clikcorr also allows the user to use other optimization methods implemented in "optim" or use the non-linear minimization function "nlm" (R Core Team, 2013a). For example:

R> clikcorr (owlData, "feather_L", "feather_U", "AT_L", "AT_U", cp=.95, dist="t", $+\mathrm{df}=3$, method="BFGS")

R> clikcorr (owlData, "feather_L", "feather_U", "AT_L", "AT_U", $c p=.95$, dist=" $t$ ", $+\mathrm{df}=3$, nlm=TRUE)

We note here that the default optimization method in "optim" function does not use derivative (or gradient) information but rather does a greedy simplex search on the function values, which makes it work relatively slowly. In future work, we plan to implement some independent gradient-directed types of optimization algorithms into clikcorr to hasten the convergence speed. It would be especially helpful in searching for the confidence interval bounds of the correlation coefficient.

clikcorr also allows R plotting options such as "xlab", "xlim", "bg" etc., in the "splot" and "plot" functions. For example, in Figure 6 the individual panels are generated respectively by

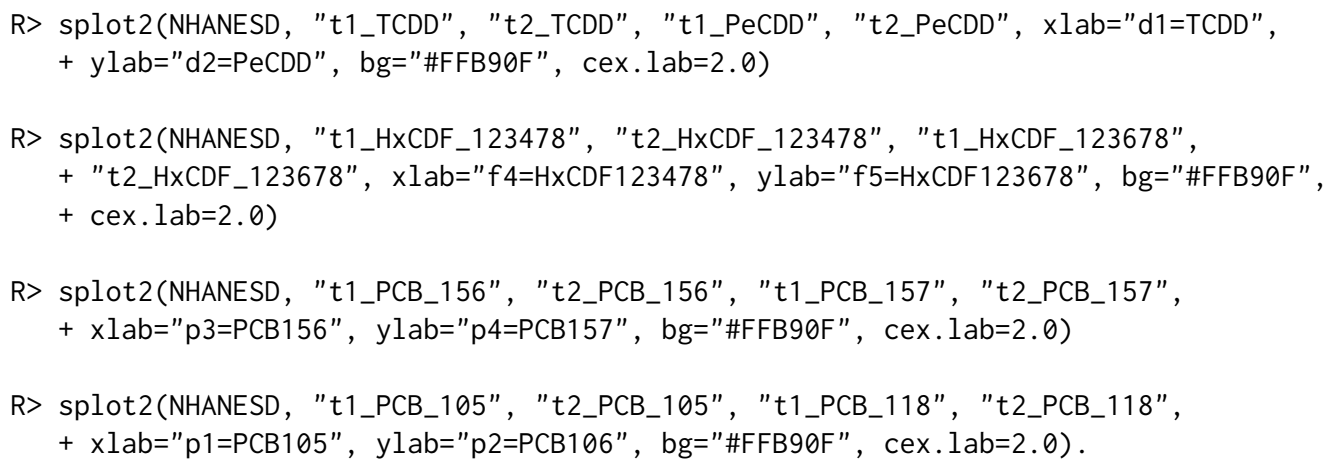



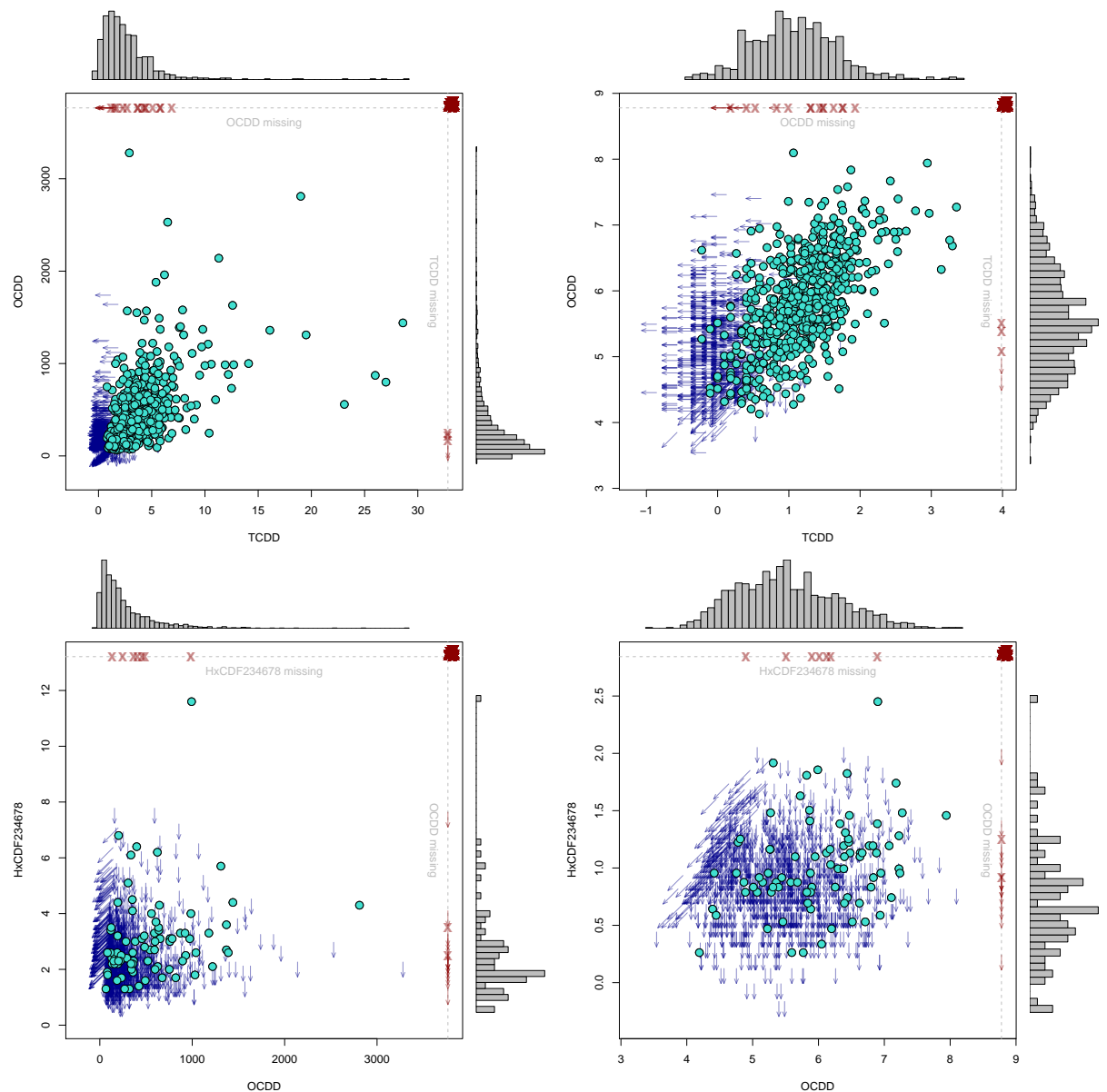

Figure 5: Example scatter plots between compound pairs with and without log transformation. Between $T C D D(\mathrm{~d} 1)$ and $O D D(\mathrm{~d} 7)$ : (a) d1-d7 without $\log$ transformation, $\hat{\rho}=0.22, p \approx 0$; (b) d1-d7 with $\log$ transformation, $\hat{\rho}=0.60, p \approx 0$. Between $O C D D(\mathrm{~d} 7)$ and $H x C D F 234678(\mathrm{f} 6)$ : (c) d7-f6 without $\log$ transformation, $\hat{\rho}=-0.05, p \approx 1$; (d) d7-f6 with $\log$ transformation, $\hat{\rho}=0.38, p \approx 0$. The histograms on top and right of each plot are marginal sample distributions from observed data. 


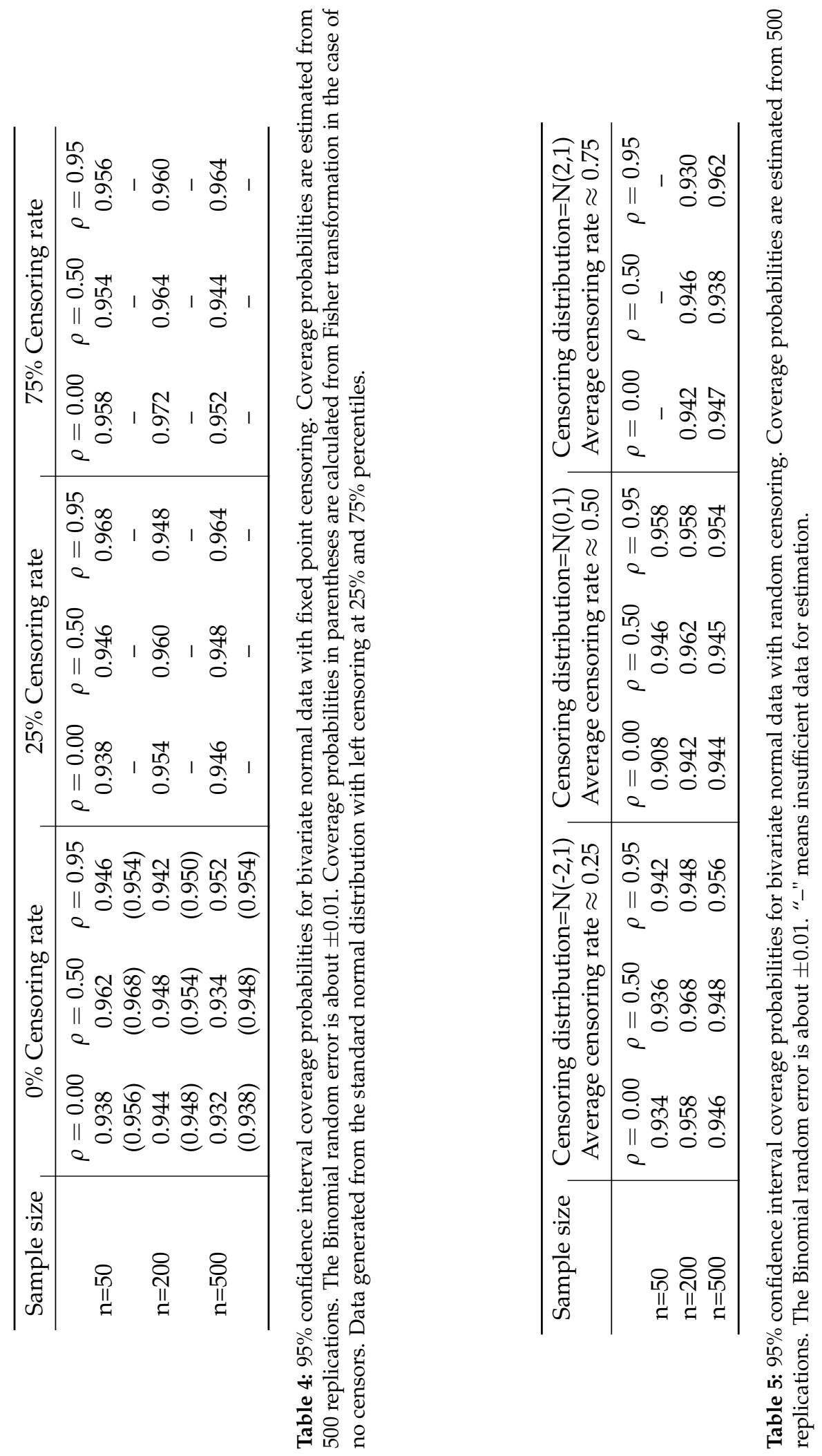




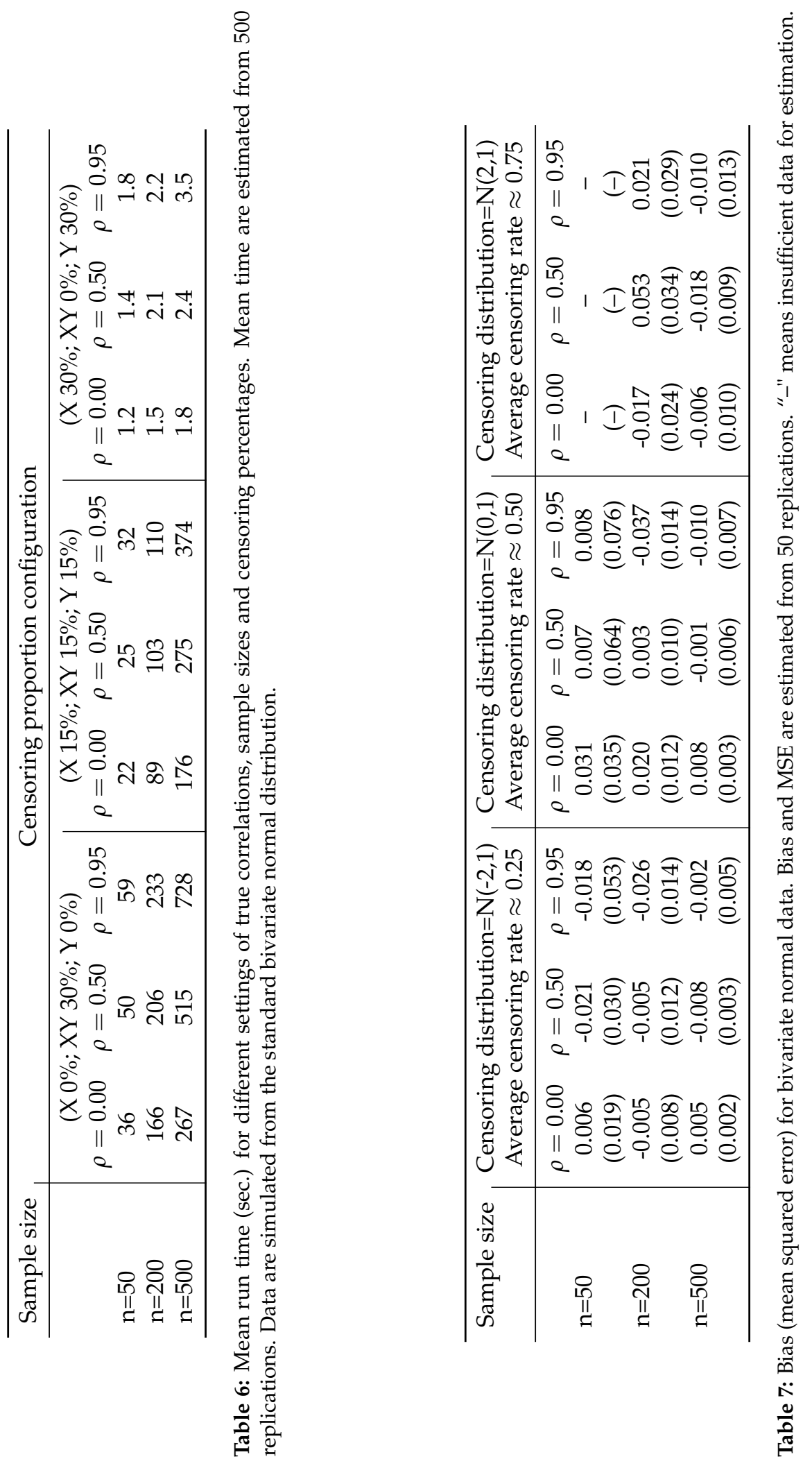




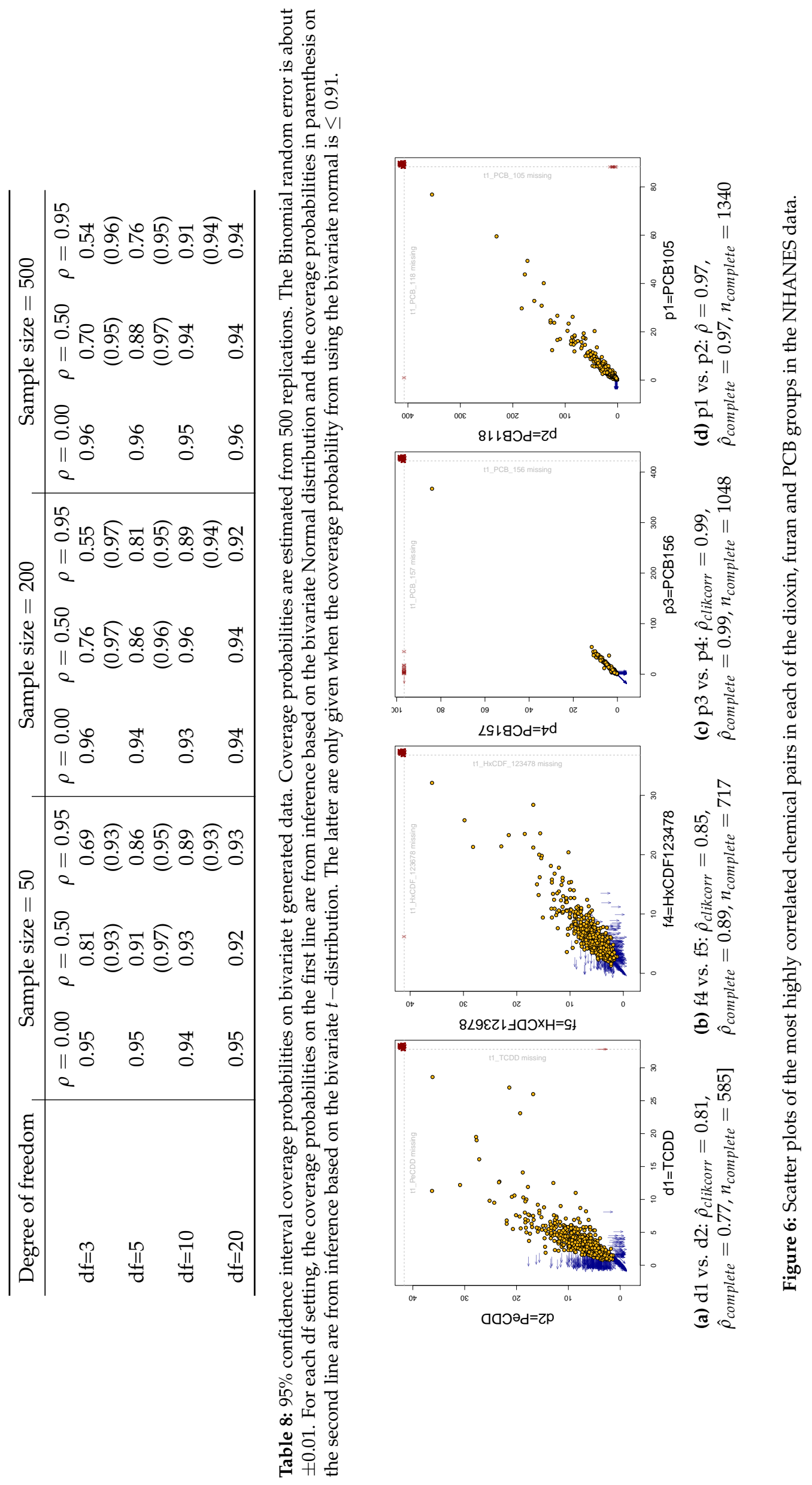




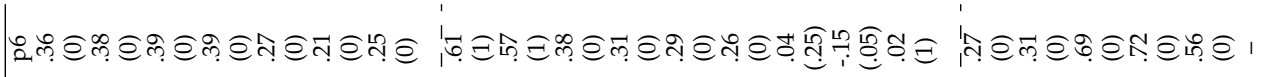

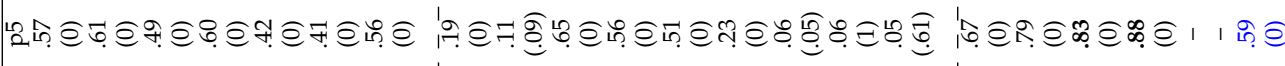

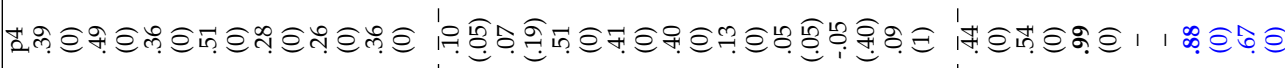

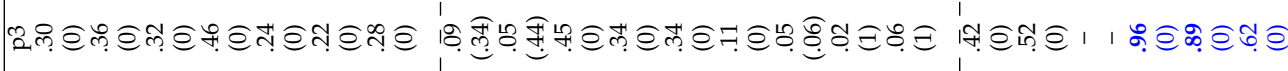
경

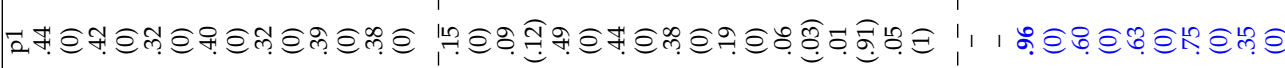

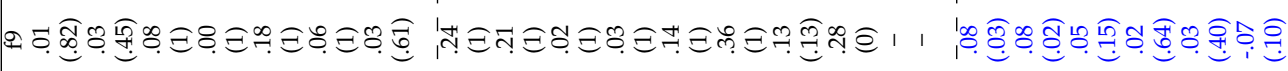

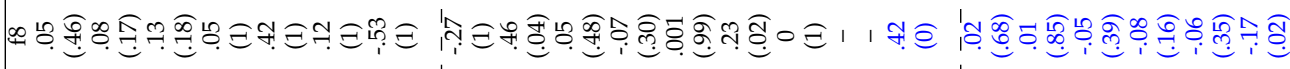

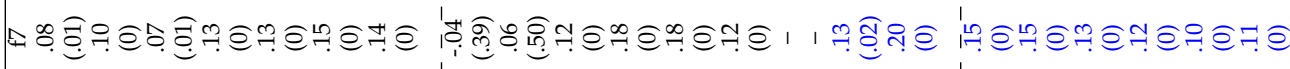
유유위

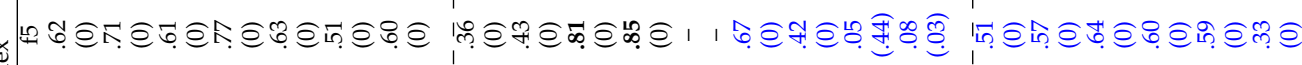
$\frac{0}{\tilde{g}}$

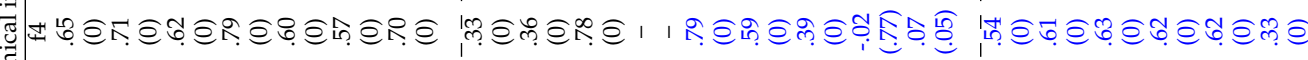

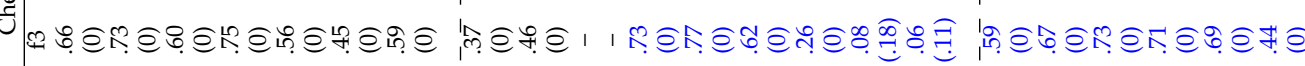

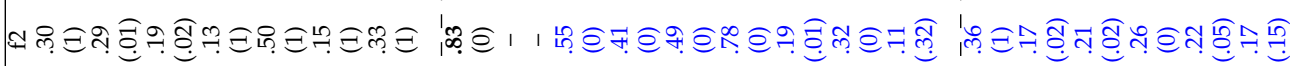

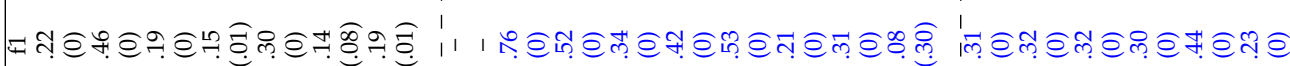
- - - - - - - - - - - -

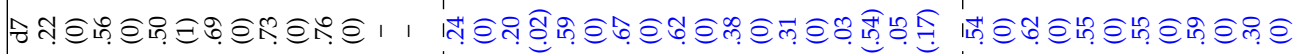
윰ํㅇำ

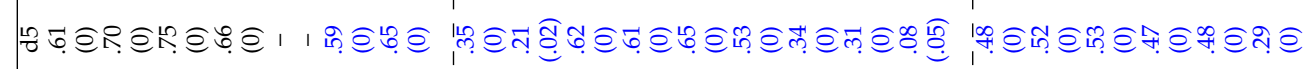

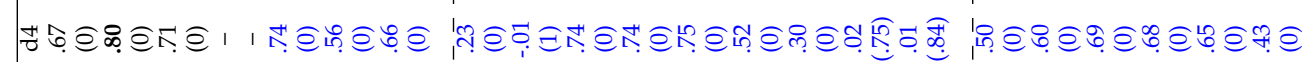

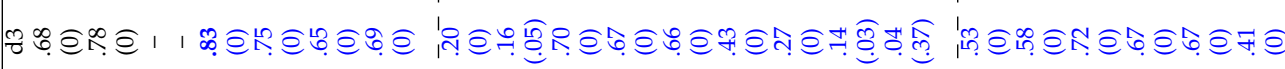
뭉 \# 1 । ㅎำ 

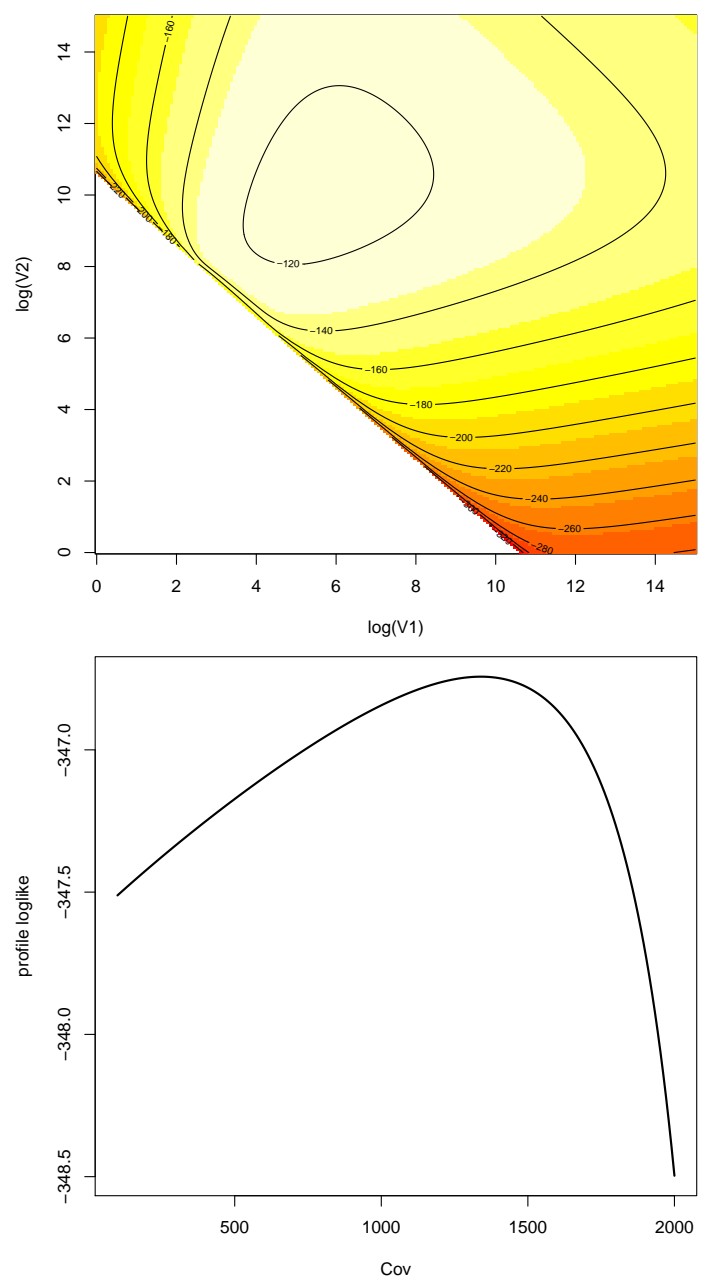

Figure 7: Illustration of effects of starting values on parameter estimates from profile log likelihood surfaces or curves. Upper panel: the profile joint log likelihood of parameters $\left(\log \left(\sigma_{X}^{2}\right), \log \left(\sigma_{Y}^{2}\right)\right)$ with the mean and correlation parameters set at certain starting values. Values from highest to lowest are colored in order of white, yellow and red; Lower panel: the profile likelihood of covariance parameter with mean and variance parameters fixed at certain starting values.

\section{Bibliography}

P. D. Allison. Survival Analysis Using SAS: A Practical Guide. SAS Institute Inc., NC USA, 2010. [p161, 163]

M. C. Bryson. Heavy-tailed distributions: Properties and tests. Technometrics, 16:61-68, 1974. URL http://dx.doi.org/10.1080/00401706.1974.10489150. [p168]

R. A. Fisher. Frequency distribution of the values of the correlation coefficient in samples of an indefinitely large population. Biometrika, 10(4):507-521, 1915. URL http://dx. doi . org/10.2307/ 2331838. [p159, 160]

A. Genz and F. Bretz. Computation of Multivariate Normal and $t$ Probabilities. Lecture Notes in Statistics. Springer-Verlag, Heideberg, 2009. ISBN 978-3-642-01688-2. [p165]

A. Genz, F. Bretz, T. Miwa, X. Mi, F. Leisch, F. Scheipl, and T. Hothorn. motnorm: Multivariate Normal and $t$ Distributions, 2012. URL http://CRAN. R-project.org/package=mvtnorm. R package version 0.9-9994. [p165]

S. R. Giolo. Turnbull's nonparametric estimator for interval-censored data. Technical Report, 2004. URL http://www.est.ufpr.br/rt/suely04a.pdf. [p161] 
D. R. Helsel. Nondetects and Data Analysis: Statistics for Censored Environmental Data. John Wiley \& Sons, New Jersey, 2005. [p163]

D. R. Helsel. Statistics for Censored Environmental Data Using MINITAB and R. John Wiley \& Sons, New Jersey, 2012. [p163]

T. Hothorn, F. Bretz, and A. Genz. On Multivariate $t$ and Gauss Probabilities in R, 2013. URL http: //cran.r-project.org/web/packages/mvtnorm/vignettes/MVT_Rnews.pdf. [p165]

V. L. Jaspers, D. Herzke, I. Eulaers, B. W. Gillespie, and M. Eens. Perfluoroalkyl substances in soft tissues and tail feathers of belgian barn owls using statistical methods for left censored data to handle non-detects. Environment International, 52:9-16, 2013. URL https://doi .org/10.1016/j . envint.2012.11.002. [p159, 160, 168]

L. Li, W. Wang, and I. Chan. Correlation coefficient inference on censored bioassay data. Journal of Biopharmaceutical Statistics, 15(3):501-512, 2005. URL https://doi .org/10.1081/BIP-200056552. [p159, 160, 163]

Y. Li, K. Shedden, B. W. Gillespie, and J. A. Gillespie. clikcorr: Censoring Data and Likelihood-Based Correlation Estimation, 2016. URL https://cran.r-project.org/web/packages/clikcorr/. [p159, 163]

R. J. A. Little and D. B. Rubin. Statistical Analysis with Missing Data. John Wiley \& Sons, New York, 2002. [p161]

S. A. Murphy and A. W. van der Vaart. On profile likelihood. J. Amer. Statist. Assoc, 95:449-465, 2000. URL https://doi .org/10.1080/01621459.2000.10474219\{"\}. [p163]

R Core Team. Non-Linear Minimization. The R Stats Package, 2013a. URL http: //ugrad. stat. ubc.ca/ R/library/stats/html/nlm.html. [p171]

R Core Team. General-Purpose Optimization. The R Stats Package, 2013b. URL http://stat. ethz.ch/Rmanual/R-devel/library/stats/html/optim.html. [p171]

C. R. Rao. Linear Statistical Inference and Its Applications. John Wiley \& Sons, New York, 1973. [p161]

S. I. Resnick. Heavy-Tail Phenomena Probabilistic and Statistical Modeling. Springer-Verlag, New York, 2007. [p168]

M. Schemper, A. Kaider, S. Wakounig, and G. Heinze. Estimating the correlation of bivariate failure times under censoring. Stat Med., 32(27):4781-4790, 2013. URL https://doi .org/10. 1002/sim. 5874. [p163]

H. Stryhn and J. Christensen. Confidence intervals by the profile likelihood methods, with applications in veterinary epidemiology. Proceedings of the 10th International Symposium on Veterinary Epidemiology and Economics, pages 208-211, 2003. [p163]

T. M. Therneau. A Package for Survival Analysis in S, 2015. URL https://CRAN.R-project.org/ package=survival. version 2.38. [p163]

D. J. Venzon and S. H. Moolgavkar. A method for computing profile-likelihood-based confidence intervals. Appl. Statist., 37(1):87, 1988. URL https://doi .org/10.2307/2347496. [p163]

H. Zhou, Z. Liao, S. Liu, W. Liang, X. Zhang, and C. Ou. Comparison of three methods in estimating confidence interval and hypothesis testing of logistic regression coefficients. Journal of Mathematical Medicine, 25(4):393-395, 2012. [p163]

Yanming $\mathrm{Li}$

Department of Biostatistics

University of Michigan

Ann Arbor, MI 48109-2029

E-mail: liyanmin@umich.edu

Brenda Gillespie

Department of Biostatistics

University of Michigan

Ann Arbor, MI 48109-2029

E-mail: bgillesp@umich. edu 


\section{Kerby Shedden}

Department of Statistics

University of Michigan

Ann Arbor, MI 48109-1107

E-mail: kshedden@umich.edu

John Gillespie

Department of Mathematics and Statistics

University of Michigan-Dearborn

Dearborn, MI 48128

E-mail: jgillesp@umich.edu 